\title{
ON THE NORMALIZER OF CERTAIN SUBALGEBRAS OF GROUP-MEASURE FACTORS
}

\author{
BY JUDITH PACKER
}

\begin{abstract}
We study operator algebras constructed from ergodic actions of discrete groups on compact Lebesgue spaces. We show that under appropriate conditions, the normalizer of a subalgebra corresponding to a quotient action depends on the relative elementary spectrum of the action over the quotient action. Using these results and methods of groupoid cohomology we prove the existence of an uncountable family of Cartan subalgebras of the hyperfinite II $_{1}$ factor, no two of which are inner conjugate.
\end{abstract}

1. Introductory remarks. Let the countable discrete group $G$ act freely and ergodically on the compact Lebesgue space $(X, \mu)$ so as to leave the finite measure $\mu$ quasi-invariant. By the von Neumann-Murray group-measure construction we then may form the factor $F(X, G)$. Let $(Y, \nu, G)$ be a quotient action of $(X, \mu, G)$; by this we mean that there exists a surjective Borel map $\varphi: X \rightarrow Y$ satisfying $\varphi^{*}(\mu)=\nu$ and $\varphi(x g)=\varphi(x) g(\mu$ a. e.) for every $g$ in $G$; we also call $(X, \mu, G)$ an extension of $(Y, \nu, G)$. Throughout the following we will assume that in the decomposition of $\mu$ over the fibers of $\varphi$ as $\mu=\int \mu_{y} d \nu, \mu_{y g}=g^{*}\left(\mu_{y}\right)$. This implies that there exists a $G$-invariant conditional expectation of $L^{\infty}(X)$ onto $L^{\infty}(Y)$. The map $\varphi$ provides a homomorphism of the ergodic measure groupoid $X \times G$, onto the groupoid $Y \times G$, and one thus obtains an injection of $F(Y, G)$ into $F(X, G)$, which we shall denote by $\varphi^{*}$. Since $G$ acts ergodically on $(X, \mu)$ it follows that $G$ acts ergodically on $(Y, \nu)$, and if $G$ acts freely on $(Y, \nu)$ as well, $F(Y, G)$ injects naturally as a subfactor of $F(X, G)$.

THEOREM 1. Let $(Y, \nu, G)$ be a free quotient action of $(X, \mu, G)$ where $X$ and $Y$ are compact Lebesgue spaces and $\mu$ (hence $\nu$ ) is finite and $G$-invariant, and where the countable discrete group $G$ acts freely and ergodically on $(X, \mu)$. Then there is a canonical correspondence between the intermediate subalgebras of $F(Y, G)$ and $F(Y, G)$ and the quotient actions $(Z, G)$ of $(X, G)$ which are extension actions of $(Y, G)$.

REMARK 1. Since $(Z, G)$ will inherit ergodicity from $(X, G)$ and freeness from $(Y, G)$, it follows immediately from the theorem that any intermediate subalgebra, which will be of the form $F(Z, G)$ for some $(Z, G)$, will be a subfactor.

Received by the editors April 1, 1982 and, in revised form, June 7, 1982 1980 Mathematical Subject Classification. Primary 46L40, 46L10, 28D99; Secondary 47C1 5, 47D99. 
REMARK 2. In Mackey's virtual group terms, if we regard $(X, G)$ as corresponding to a virtual subgroup $V_{X}$ of $G$, then $(Y, G)$ corresponds to a virtual subgroup $V_{Y}$ of $G$ which "contains" $V_{X}$. The subalgebras in between $F(X, G)$ and $F(Y, G)$ correspond to the virtual subgroups lying "in between" $V_{Y}$ and $V_{X}$. See [5] for details about virtual groups.

2. Relatively elementary spectrum and the normalizers of subfactors. Let $(X, \mu, G)$ and $(Y, \nu, G)$ be as in the previous section, so that $\mu$ and $\nu$ are finite measures, $\mu$ relatively $G$-invariant over $\nu$, and $(Y, G)$ is a quotient action of $(X, G)$, where $(X, \mu, G)$ is ergodic. Then we can decompose $L^{2}(X, \mu)$ as a direct integral $\int_{Y}^{\oplus} H_{y} d \nu$ where $H_{y}=L^{2}\left(\varphi^{-1}(y), \mu_{y}\right)$. Since we have stipulated that $\mu_{y g}=g^{*}\left(\mu_{y}\right), \nu$ a. e. for every $g$ in $G$, we have a unitary mapping $\alpha(y, g): H_{y g}$ $\rightarrow H_{y}$ for $(y, g)$ in $Y \times G$ given by $\alpha(y, g)(f)(x)=f(x g)\left(x \in \varphi^{-1}(y)\right)$. Furthermore $\alpha\left(y, g_{1} g_{2}\right)=\alpha\left(y, g_{1}\right) \alpha\left(y g_{1}, g_{2}\right)$. Following Zimmer [11] we call $\alpha$ a bundle cocycle representation, and say that $(X, \mu, G)$ has relatively discrete (respectively relatively elementary) spectrum over $(Y, \nu, G)$ if the above cocycle representation decomposes as the direct sum of finite-dimensional (respectively one-dimensional) subbundle representations. Zimmer in [11] showed that if $(Y, \nu, G)$ is a quotient action of the ergodic action $(X, \mu, G)$ where $\mu$ and $\nu$ are finite and $G$-invariant, then there exists a maximal extension of $(Y, G)$ and quotient action of $(X, G)$ having relatively discrete (respectively relatively elementary) spectrum over $(Y, G)$. (This holds true as well when $\mu$ and $\nu$ are finite and we only have relative invariance of $\mu$ over $\nu$.) Call $\left(Z_{e}, G\right)$ the maximal extension with relatively elementary spectrum over $(Y, G)$.

THEOREM 2. Let $(Y, \nu, G)$ be a free quotient action of the ergodic action $(X, \mu, G)$, with $\mu$ finite, and relatively $G$-invariant over $\nu$, where $G$ is a countable discrete group. Then the subalgebra of $F(X, G)$ generated by the unitary elements of $F(X, G)$ which normalize $F(Y, G)$ is equal to $F\left(Z_{e}, G\right)$.

The proof of Theorem 2 follows from examining the fiber product $G$ space $\left(X \times_{Y} X, G\right)$, where $X \times_{Y} X=\left\{\left(x_{1}, x_{2}\right) \in X \times X \mid \varphi\left(x_{1}\right)=\varphi\left(x_{2}\right)\right\}$ and $\left(x_{1}, x_{2}\right) g=$ $\left(x_{1} g, x_{2} g\right)$. This action will be free, but need not be ergodic. Define $\varphi_{1}\left(\left(x_{1}, x_{2}\right)\right)$ $=x_{1} ; \varphi_{2}\left(\left(x_{1}, x_{2}\right)\right)=x_{2}$. Then $\left(\varphi \circ \varphi_{2}\right)^{*}=\left(\varphi \circ \varphi_{1}\right)^{*}$ embeds $F(Y, G)$ into $F\left(X \times_{Y} X, G\right)$, and the maps $\varphi_{1}^{*}$ and $\varphi_{2}^{*}$ give two embeddings of $F(X, G)$ into $F\left(X \times_{Y} X, G\right)$. If $U \in F(X, G)$ normalizes $F(Y, G)$, one uses the fact that $\varphi_{1}^{*}(U) \varphi_{2}^{*}\left(U^{*}\right)$ commutes with everything in $\varphi_{1}^{*} \varphi^{*}(F(Y, G))$ to show that $U$ is of the form desired.

A natural question to ask is what happens when $(Y, v, G)$ is not free (and thus $F(Y, G)$ need not be a factor). The simplest example of this is the case ( $Y=$ pt., $G$ ), the trivial quotient action of $G$. If we embed $F($ pt., $G)$ into $F(X, G)$ 
we obtain a subalgebra which we shall denote by $S(X, G)$ which is maximal abelian if $G$ is abelian and $(X, G)$ is ergodic. Dixmier in [2], introduced a partial classification of maximal abelian subalgebras in a factor by defining the following categories.

Definition [2]. Let $A$ be a maximal abelian subalgebra of the factor $M$. Let $N(A)$ be the von Neumann subalgebra of $M$ generated by $\{U$ unitary in $\left.M \mid U^{*} A U=A\right\}$. Then $A$ is called (regular, semiregular, singular) in $M$ if $N(A)$ is (all of $M$, a subfactor of $M$, only $A$ ), respectively.

The following theorem is implicit in Hahn [4], though not formulated as below, and is clearly related to Theorem 2 . We conjecture that one can remove the freeness hypothesis from Theorem 2 for $G$ abelian by somehow combining its proof with the proof of the theorem below.

THEOREM 3 (see [4]). Let $G$ be a countable discrete abelian group and let $(X, \mu)$ be a compact Lebesgue space on which $G$ acts freely and ergodically and in such a way as to preserve the finite measure $\mu$. Then $N(S(X, G))$ is equal to $F\left(Y_{d}, G\right)$, where $\left(Y_{d}, G\right)$ is the maximal quotient action of $(X, G)$ with pure point spectrum.

For $(X, \mu, G)$ as in Theorem 3, we have been able to construct all of the unitaries in $F(X, G)$ which normalize $S(X, G)$ by extending methods used by Singer in [9] and those used by Takesaki in [10]. When $G$ is discrete but not abelian, other methods must be used. The following theorem was first proved by $O$. A. Nielsen for the case $G$ abelian, using similar to those used in $[4,10]$. We have reproved the theorem using different techniques for $G$ nonabelian as well.

THEOREM 4. Let the countable discrete group $G$ act freely and ergodically on the compact Lebesgue space $(X, \mu)$ in such a way as to preserve the finite measure $\mu$ and suppose that the unitary representation of $G$ on $L^{2}(X, \mu) \ominus \mathbf{C}$ has no finite-dimensional subrepresentations $(i . e .(X, \mu, G)$ has continuous spectrum). Then any unitary in $F(X, G)$ which normalizes $S(X, G)$ must lie in $S(X, G)$.

In the attempt to classify maximal abelian subalgebras (m.a.s. for short) of factors, an important invariant is the length of an m.a.s. If $A$ is an m.a.s. of the factor $\mathrm{M}$, define $\mathrm{A}=N_{0}$, and proceed inductively, setting $N_{i+1}=N\left(N_{i}\right)$. If $\eta$ is a limit ordinal, set $N_{\eta}=$ the von Neumann algebra generated by $\bigcup_{\xi<\eta} N_{\xi}$. Then $N_{\eta}$ is well defined for any countable ordinal $\eta$, and if $\xi \leqslant \eta$, $N_{\xi} \subseteq N_{\eta}$. Define the length of $\mathrm{A}$ in $\mathrm{M}$ to be the least countable ordinal $\eta$ such that $N_{\eta}=N_{\eta+1}$, if such an ordinal exists. The following is a corollary of Theorems 2 and 3. 
COROLLARY. Let $(X, \mu, G)$ be a free ergodic action of the countable discrete abelian group $G$ on the compact Lebesgue space $X$ preserving the finite measure $\mu$, such that the maximal quotient action of $(X, \mu, G)$ with pure point spectrum is a free action. Suppose that the length of $S(X, G)=\eta$. Then $N_{\eta}=F(Z, G)$ where $(Z, G)$ is the maximal quotient action of $(X, G)$ with generalized elementary spectrum, and $\eta$ is equal to the ordinal of this spectrum. (See [11] for definitions.)

REMARK. If $G$ is equal to $\mathbf{Z}$, then the maximal quotient action of $(X, \mathbf{Z})$ with discrete spectrum is either free or transitive. If transitive, then $(X, Z)$ will have relatively continuous spectrum over it. We thus may always compute the length of $S(X, Z)$ in $F(X, Z)$ if the action of $\mathbf{Z}$ preserves the measure $\mu$.

Other results in this line which we hope to publish soon include a correspondence between Zimmer's notion of normal extensions of ergodic actions with relatively discrete spectrum [11] and Nakagami's notion of crossed dual products of von Neumann algebras by compact groups [6].

3. Examples and applications. Let an action of $Z$ on the circle $S^{\mathbf{1}}$ be generated by rotation through an angle which is an irrational multiple of $2 \pi$. The corresponding $S\left(S^{\mathbf{1}}, \mathrm{Z}\right)$ is then regular by Theorem 3. If $\beta: S \times \mathrm{Z} \rightarrow S^{\mathbf{1}}$ satisfies the cocycle identity $\beta\left(z, n_{1}+n_{2}\right)=\beta\left(z, n_{1}\right) \beta\left(z n_{1}, n_{2}\right)$, then by [9] $\beta$ gives rise to a ${ }^{*}$-automorphism of $F\left(S^{1}, Z\right)$ which can be extended to a ${ }^{*}$-automorphism of $F(X, Z)$ for $(X, Z)$ any extension action of $\left(S^{\mathbf{1}}, Z\right)$. We denote this automorphism by $A_{\beta}$.

Proposition. The regular maximal subalgebra $A_{\beta}\left(S\left(S^{1}, Z\right)\right)$ is inner conjugate to $S\left(S^{1}, Z\right)$ in $F\left(S^{1}, Z\right)$ if and only if there exists a measurable function $f: S^{1}$ and $\lambda$ in $S^{1}$ such that $\beta(z, n)=f(z n) \lambda^{n}(f(z))^{-1}(*)$.

The proof of the proposition follows upon finding the minimal extension of $\left(S^{1}, Z\right)$, say $(X, Z)$, such that $A_{\beta}$ is an inner automorphism of $F(X, Z)$, and then applying Theorem 3.

Since a $\mathrm{II}_{1}$ factor possesses a faithful normal conditional expectation onto each of its von Neumann subalgebras, any regular maximal abelian subalgebra of the hyperfinite $\mathrm{II}_{1}$ factor is in fact a Cartan subalgebra. In [3] Feldman and Moore proved the existence of two noninner conjugate Cartan subalgebras of the hyperfinite $\mathrm{II}_{1}$ factor by using the method of contradiction. The referee has kindly pointed out to us that a relatively simple extension of the argument of [3] shows that the set of inner conjugacy classes of Cartan subalgebras of the hyperfinite $\mathrm{II}_{1}$ factor is uncountable. We have

THEOREM 5. There exists an uncountable family of Cartan subalgebras of the hyperfinite $\mathrm{II}_{1}$ factor, contained in the set $\left\{A_{\beta}\left(S\left(S^{1}, \mathrm{Z}\right)\right) \mid \beta: S^{1} \times \mathrm{Z} \rightarrow S^{1}\right.$ a cocycle\}, no two of which are inner conjugate. 
One proves the theorem by finding an uncountable family of cocycles for $\left(S^{1}, Z\right)$ with values in $S^{1}, F$, such that $\beta_{1}, \beta_{2} \in F, \beta_{1}\left(\beta_{2}\right)^{-1}$ satisfies $(*)$ implies $\beta_{1}=\beta_{2}$. Then $\left\{A_{\beta}\left(S\left(S^{1}, Z\right)\right) \mid \beta \in F\right\}$ is the required family, by the proposition.

We are indebted to Professor George Mackey for many helpful conversations.

\section{REFERENCES}

1. J. Dixmier, Sous-anneaux abeliens maximaux dans les facteurs de type fini, Ann. of Math. (2) 59 (1954), 279-286. 1957.

2. - Les algèbres d'opérateurs dans l'espace Hilbertien, Gauthier-Villars, Paris,

3. J. Feldman and C. C. Moore, Ergodic equivalence relations, cohomology, and von Neumann algebras. II, Trans. Amer. Math. Soc. 234 (1977), 325-359.

4. P. Hahn, Reconstruction of factors from measures on Takesaki's equivalence rela. tion, J. Funct. Anal. 31 (1979), 263-271.

5. G. Mackey, Ergodic theory and virtual groups, Math. Ann. 166 (1966), 187-207.

6. Y. Nakagami, Dual action on a von Neumann algebra and Takesaki's duality theorem for a locally compact group, Publ. R. I. M. S. Kyoto Univ. 12 (1977), 727-775.

7. O. A. Nielsen, Maximal abelian subalgebras of the hyperfinite factor, II, J. Funct. Anal. 6 (1970), 192-202.

8. J. Renault, $A$ groupoid approach to $C^{*}$-algebras, Lecture Notes in Math., vol. 793, Springer-Verlag, Berlin and New York, 1980.

9. I. Singer, Automorphisms of finite factors, Amer. J. Math. 77 (1955), 117-133.

10. M. Takesaki, On the unitary equivalence among the components of decomposition of representation of involutive Banach algebras and the associated diagonal algebras, Tôhoku Math. J. 25 (1963), 365-393.

11. R. Zimmer, Extensions of ergodic actions and generalized discrete spectrum, Illinois J. Math. 20 (1976), 373-409; 555-588.

DEPARTMENT OF MATHEMATICS, HARVARD UNIVERSITY, CAMBRIDGE, MASSACHUSETTS 02138 
